\title{
Novel Cartographical Designs for Blind and Partially Impaired Students in Kurdistan
}

Ashna Abdulrahman Kareem Zada / Eötvös Loránd University / Faculty of Informatics Department of Cartography and Geoinformatics/ ashnakareem88@gmail.com

\begin{abstract}
It is understood that all human beings need information, including spatial (geospatial) information. It has to be noted that spatial and geospatial information helps people to enrich the knowledge about certain regions. In general, the knowledge is very significant for strengthening orientation ability, which, in turn, affects the level of intelligence. It is believed that the better a person can recognize geographical information the cleverer s/he is. Being able to have the spatial knowledge helps humans to fulfil their basic needs and find solutions to various problems. Present research uses Geographical Information System (GIS) to create paper maps, which can be transferred onto a touch display where the user can choose different keys and settings (such as colour, measurement, etc.) to represent different forms of data. The study aims to examine the effectiveness of digital interactive cartographical novel solutions in improving text and space memorization among visually impaired Kurdish students. The study's purpose draws from the knowledge that Kurdistan learners and other demographic groups lack an effective multimodal communication map suited to contemporary society's demands. The research seeks to fill the gap by presenting a strategy for developing interactive tactile maps for the target group to solve the growing problem of visual impairment in Kurdistan. Technologies associated with tactile maps are reviewed and the application of interactive tactile maps for populations, in addition to those who are visually impaired, is also considered. The obtained results are very useful for blind and low sighted users to communicate information through the assistive technologies on the map due to finding the geographical location easily.
\end{abstract}

Keywords: Tactile Maps, GIS, Sightless, Education

\section{Introduction}

Blind and partially impaired students suffer from disproportionate effects from the ill-equipped Iraqi educational sector that does not consider their specialized needs. Such students require continuous support through their schooling years. The support includes training and instruction from teachers specialized in working with the visually impaired. However, this population demographic continues to attract the attention of stakeholders. Mowfaq (2019) noted that dozens of blind and partially impaired learners graduate from school every year in the Kurdistan region. The leading stakeholder in these successes is the Runaki Institute, which implemented the braille system to enable learners to read news, bulletins and other subjects. Graduates and analysts concede that braille is an excellent strategy for people with special needs (Mowfaq, 2019). As a result, stakeholders explore opportunities for the integration of braille into all the dimensions of learning. One area of particular interest for educators and blind and partially impaired students is applying the novel pedagogical solutions to cartography.

Finding appropriate new technological learning tools for blind students is the objective of educators across the board. Ahmed (2021, p. 51) asserted that the main problem blind people face in today's technologically advanced world is the technology itself. Leaners with visual impairment use smart versions of auditory devices. The gadgets have screen readers that convert optical and diagrammatic data into an audible version without considering the context. The outcome is inefficient in using the devices to operate independently. According to
Ahmed (2021), the Kurdish visually impaired people encounter numerous hurdles getting an education and using smart gadgets, a situation compounded by discriminatory practices in the educational sector.

Concerted efforts focus on using the map to provide geographical information for the Kurdish blind and partially impaired population. Existing research on the subject focuses on applying the geographic information system (GIS) to create paper maps transferable to touch displays. The tactile feature allows the user to choose different keys and settings for coding elements, such as colour and measurement, representing diverse forms of data (Zada, 2020).

Researchers applied the test to visually impaired people in Kurdistan, Iraq, to apprehend the level to which they use paper maps. Similarly, researchers sought to establish the reasons behind some professionals' reluctance to use the new technology. The implication was the exploration of the opportunity for introducing tactile maps into the school curriculum. Therefore, the study attempts to validate novel cartographical strategies for visually impaired learners in the Kurdistan region.

\section{Findings and discussion}

As a state that the goal of the research is to create efficient maps to aid the Iraqi Kurdistan education in implementing GIS to improve their curriculums, so the main aim was to find out to what extent blind and visually impaired people achieve the positive aspects of mapping. Therefore, the research is empirical and consists of two main directions. The first direction includes traditional solutions and the second one modern 
(computer-based) solutions. These are two main approaches to the methodology, because both are the basis and start point for the development of the modern ones. The comparison between the tradition and digital maps proves beyond reasonable doubt that there is an advanced approach used in digital maps to cater to the partially blind individuals studying cartography (Real and Araujo, 2019). The digital maps are more efficient since they offer room for the analysis and altering the data in the maps that require updating in the system. This can be achieved through the development and analysis using the underlying ArcGIS software, which can develop more efficiently thematic content for maps than using traditional methods. On the other side, the paper-based maps do not offer room for manipulations and updating the latest trends and contextualization of the data, such as the graphics and the images contained to the current status view (Zada, 2019).

As a result of the underlying hypothetical facts, the individuals in the region think that the digital maps are more essential than the old traditional maps that lack the latter's advantages. Besides, the assertion can be confirmed since digital maps are more beneficial than traditional maps since they offer better technicalities such as the ideal service to visually impaired individuals; the digital mapping technology, the visually impaired individuals, can offer their senses be touchable. However, the low vision people, more so the partially impaired people in the community, cannot use both maps properly since they are not capacitated to perform the tasks effectively. This incapacitation can be observed through the results obtained when visually impaired individuals use paper and digital maps exclusively. Almost everyone in the community faces a challenge in using digital and paper maps depending on the severity of the impairment. The reasoning behind the challenge in the utilization of the mapping in most cases is associated with the insufficiency in the computers and other mobile technologies (Jiang, 2020). Besides the loss of the batteries and the other problems are associated with the utilization of other technologies which are linked with the incapacity in performing tasks such as the utilization of the digital maps by the blind or partially impaired individuals. On the other side, the utilization problem is immense with the increased incompetence in using the computer map platforms, such as implementing the low vision apps essential to offer specialized services in Kurdistan for the people with low vision impairment.

\section{Impact of the ArcGIS in Mapping}

Maps are the most significant solution in offering geographical data for visually impaired persons. Current cartographers utilize Geographical Information Systems (GIS) to develop paper maps that can be modified into touch display, where the visually impaired individuals can opt for various settings and keys, for instance, measurement and color to represent various data forms (Gillings, 2017, p. 122).

ArcGIS plays a vital role in developing the links between the paper map and the digital map in the cartographic studies more in the people with partial visually impairments in the society. ArcMap is essential since it symbolizes the need to collect the geographical information in one place, providing a map with a collection of layers. Furthermore, this software's technicality comes with the essential elements of the map (International Journal of Cartography, 2017). The results from the application of the ArcGIS program illustrate the importance of the need for the proper understanding of the impact of the language on the performance of the maps in the region. The students and other stakeholders need to choose the ideal language to incorporate in the maps. It is proven that the language is bringing a challenge in understanding the ArcGIS impact in the formulation of the digital maps, to enhance the visually impaired people to understand and study the maps. The most favourable language, which proved to be more familiar among people with vision impairment, is English. Hence, the English language is favourable in the generation of braille.

Most people prefer learning by the school to get convinced on the proper understanding of the mapping technicality of the Geographical Information Systems. People do not prefer learning the software at home since they lack the proper understanding of technicality; hence need to be taught by specialized people at the learning institutions, which offer the knowledge on ArcGIS software. By illustration of this motive, it is clear that ArcGIS has made people flood in learning institutions to obtain the technicality to utilize the software; besides obtaining this information, they would be able to improve the mapping technology. This is the main way to learn the braille system and the entire mapping, which makes the vision impaired individuals prefer the digital way to learn the mapping system than the traditional way of map use. The result from comparing both versions were that students preferred the new modern maps and rejected the old one made by hand as it shows in both Figures 1 and 2 . Most of the students were satisfied with the new available technology in their schools for learning and using maps.

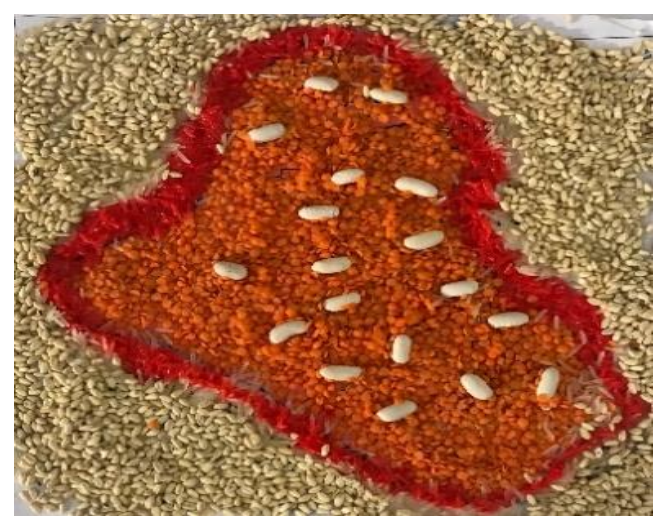

Figure 1: One of the handmade maps created by the students of the Runaki Center. 


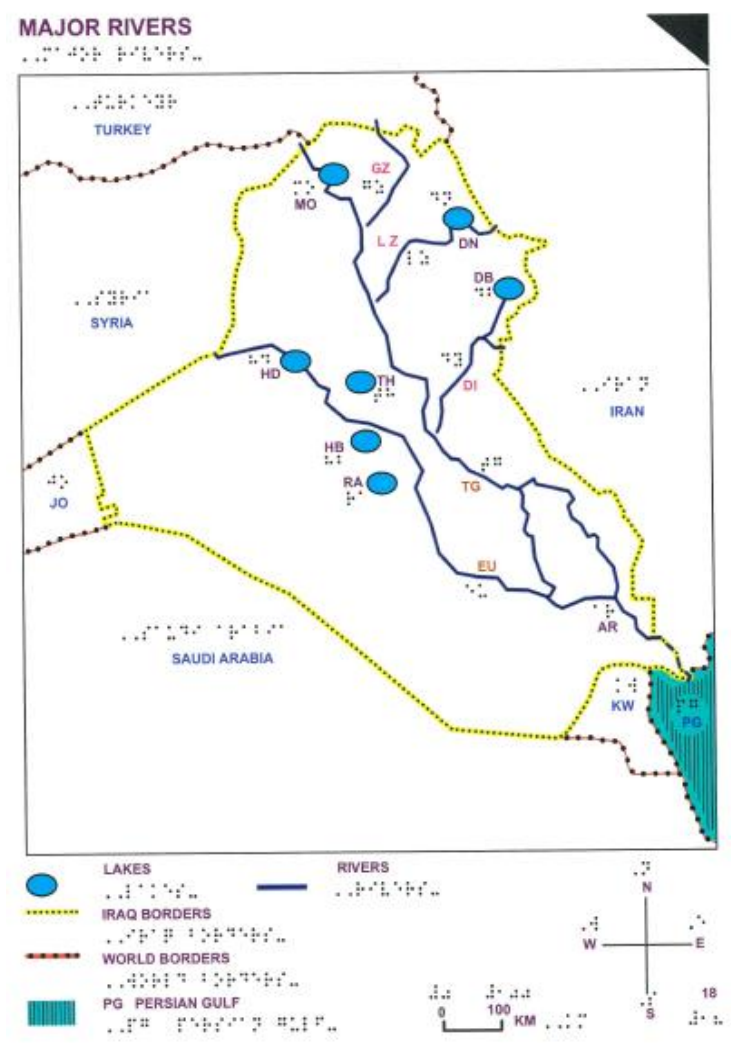

Figure 2: One of the modern maps created during this research for the Runaki Center.

\section{Conclusion}

This research is essential in coming up with new solutions in using cartography for blind and partially impaired students in Kurdistan. There has been increasing development in cartography, more so from the $21 \mathrm{st}$ century, which has brought new solutions to the underlying challenges faced by visually impaired students in the utilization of maps. Mapping requires proper vision since the intention of the students is to not the specific features in the maps hence need for good visual ability. However, there is an increase in the advancement of the technology, which is perceived to be in line with making strides to enable the visually impaired individuals to participate in the utilization of the mapping technicalities. Digital maps have come up with vast advantages in utilizing the maps than the traditional paper maps because they cater to other technologies that are essential to enable visually impaired individuals to work in the mapping system. Arc Map software has proved to be the best multimodal tool in creating a map template that all people can access. This method incorporates the use of other GIS methods in the utilization of the mapping. This method offers the quality outcomes of the mappings, such as the simplified and smoothing tools for the creation of the ArcMap boundaries and the generation of the physical features in the maps. All these tools require proper understanding of the language in the implementation to ease the utilization of the maps by visually impaired individuals. For instance, the braille system in this region should be produced in English language to be utilized by all populations. In summary, this paper proposes the use of the braille tactile maps, which are essential in enhancing the functionality of the blind or the partially blind students, such as the provision of ease access and the utilization of the maps. These maps are essential since they can provide easy updating frameworks since the technology is ever changing; indeed, the technology is more dynamic since it is developing day by day, but the digital braille tactile maps can incorporate the innovations which come along with the print technologies. Over and above, the solution must come along with the increased staff in schools and the increment in the computers and other devices essential in the incorporation of the advanced technologies. For instance, mobile phone gadgets have enhanced the learning experience in children more so in exposing the students to the latest information and latest technological advancements.

\section{References}

1. Ahmed, S.K., 2021. Finding a new technological tool for blind people and including Kurdish language in operating systems: (A Case study on the blind people of Kurdistan region). Black Sea Journal of Management and Marketing, 2(1), pp.5562.

2. Gillings, M. (2017). Mapping liminality: Critical frameworks for the GIS-based modelling of visibility. Journal of Archaeological Science, 84, pp.121-128.

3. International Journal of Cartography. (2017). User studies in cartography: opportunities for empirical research on interactive maps and visualizations. [online] Available at: https://www.tandfonline.com/doi/full/10.1080/237293 33.2017.1288534 [Accessed 22 Apr. 2021].

4. Jiang, Y. (2020). A solution to analyze mobile eye-tracking data for user research in GI Science University of Twente Student Theses. Utwente.nl. [online] Available at: http://essay.utwente.nl/85207/ [Accessed 22 Apr. 2021].

5. Mowfaq, V. 2019. Braille helps Kurdish students see a different future. Rudaw. Available at https://www.rudaw.net/english/kurdistan/04012019. [Accessed 29 March 2021].

6. Real and Araujo (2019). Navigation Systems for the Blind and Visually Impaired: Past Work, Challenges, and Open Problems. Sensors, [online] 19(15), p.3404. Available at: https://www.ncbi.nlm.nih.gov/pmc/articles/PMC6696 419/ [Accessed 22 Apr. 2021].

7. Zada, A.A.K., 2019. Testing maps for visually impaired people in Kurdistan. In Proceedings of the ICA (Vol. 2, pp. NA-NA). Copernicus GmbH.

Zada, A.A.K., 2020. Tactile mapping in Kurdistan regions, Iraq: Understanding the rules of developing on tactile cartography design. Proceedings Vol. 1, 8th International Conference on Cartography and GIS, 2020, Nessebar, Bulgaria ISSN: 1314-0604. 